\title{
O IMPACTO DA FISIOTERAPIA NA REABILITAÇÃO PSICOSSOCIAL DE PORTADORES DE TRANSTORNOS MENTAIS ${ }^{1}$
}

\section{Soraya Batista da Silva²; Luiz, Jorge Pedrão ${ }^{3}$; Adriana Inocenti Miasso ${ }^{3}$}

\begin{abstract}
São muitos os comprometimentos corporais provenientes dos transtornos mentais e dos tratamentos somáticos. Este estudo objetivou mostrar a contribuição da fisioterapia na minimização desses comprometimentos e na reabilitação psicossocial de portadores de transtornos mentais. Utilizaram-se 24 sessões de fisioterapia, com 15 portadores de transtornos mentais que ofereceram depoimentos. Os resultados mostraram que a fisioterapia contribuiu, minimizando os comprometimentos corporais causados pelos transtornos mentais e, pelos tratamentos somáticos, promoveu benefícios físicos e psíquicos, favoreceu a interação e as relações de amizade, permitindo a conclusão de que ela, a fisioterapia, facilitou os relacionamentos e, com isso, criou mais possibilidades de reabilitação psicossocial.
\end{abstract}

Descritores: Fisioterapia (Especialidade); Reabilitação; Saúde Mental.

\footnotetext{
${ }^{1}$ Apoio financeiro do Conselho Nacional de Desenvolvimento Científico e Tecnológico (CNPq) processo no 132911/2007-8.

2 Fisioterapeuta, Mestre em Ciências. E-mail: soraya.bs@hotmail.com.

${ }^{3}$ Enfermeiros, Doutor em Enfermagem, Professor Doutor, Escola de Enfermagem de Ribeirão Preto, Universidade de São Paulo, Centro Colaborador da OMS para o Desenvolvimento da Pesquisa em Enfermagem, SP, Brasil. E-mail: Luiz -lujope@eerp.usp.br, Adriana amiasso@eerp.usp.br.
} 


\section{THE IMPACT OF PHYSICAL THERAPY IN THE PSYCHOSOCIAL REHABILITATION OF CARRIERS OF MENTAL DISORDERS}

There are many body compromises from mental disorder and somatic treatments. This study had the objective of showing the contribution of physical therapy in the minimization of these compromises in the psychosocial rehabilitation of carriers of mental disorders. A total of 24 physical therapy sessions were made, with 15 carriers of mental disorders that offered statements. The results show that the physical therapy contributed minimizing the body compromises caused by the mental disorders, and by the somatic treatments, promoted physical and psychic benefits, favored the interaction and friendly relations, allowing the conclusion that it, the physical therapy, enabled relationships, and therefore, created more possibilities of psychosocial rehabilitation.

Descriptors: Physical Therapy (Specialty); Rehabilitation; Mental Health.

\section{El IMPACTO DE LA FISIOTERAPIA EN LA REHABILITACIÓN PSICOSSOCIAL DE PORTADORES DE TRASTORNOS MENTALES}

Son muchos los comprometimientos corporales provenientes de los trastornos mentales y de los tratamientos somáticos. Este estudio objetivó mostrar la contribución de la fisioterapia en la minimización de eses comprometimientos y en la rehabilitación psicosocial de portadores de trastornos mentales. Se utilizaron 24 sesiones de fisioterapia, con 15 portadores de trastornos mentales que ofrecieron declaraciones. Los resultados mostraron que la fisioterapia aportó, minimizando los comprometimientos corporales causados por los trastornos mentales y, por los tratamientos somáticos, promovió beneficios físicos y psíquicos, favoreció la interacción y las relaciones de amistad, permitiendo la conclusión de que ella, la fisioterapia, facilitó las relaciones y, con eso, creó más posibilidades de rehabilitación psicosocial.

Descriptores: Terapia Física (Especialidad); Rehabilitación; Salud Mental.

\section{Introdução}

A participação de profissionais de embasamento corporal, como o fisioterapeuta, tem se tornado cada vez mais necessária nas equipes de saúde mental, devido à busca por terapêuticas capazes de minimizar as alterações corporais apresentadas pelos portadores de transtornos mentais. Contudo, a literatura científica acerca do tema ainda é restrita a poucos estudos ${ }^{(1-2)}$.

Dentro do conjunto de manifestações que caracterizam os transtornos mentais, ocorrem alterações na estrutura corporal e no movimento, tais como dificuldades na execução dos movimentos, tensões e rigidez muscular crônica, alterações posturais, padrão anormal de respiração e prejuízo da expressão corporal ${ }^{(3-4)}$.

A presença dessas alterações corporais, que podem acontecer em decorrência do próprio transtorno mental ou pela ação prolongada de medicamentos psicotrópicos ${ }^{(3-4)}$, interfere significativamente na realização de atividades cotidianas e nas relações interpessoais ${ }^{(1,5)}$.

Os trabalhos de intervenção corporal no campo da saúde mental têm como foco principal o retorno à realidade do corpo, tão distanciada, para o portador de transtorno psíquico $^{(2)}$. 
A fisioterapia dispõe de inúmeras possibilidades terapêuticas capazes de aprimorar a funcionalidade motora, auxiliando a reestruturação dos aspectos físicos e psíquicos do indivíduo, promovendo, assim, seu processo de reabilitação ${ }^{(2)}$.

A atuação do fisioterapeuta na equipe de saúde mental é necessária, tanto em serviços ambulatoriais e hospitalares quanto em hospitais dia, centros de convivência e de atenção psicossocial e, ainda, cooperativas de trabalho, fazendo da fisioterapia uma somatória terapêutica na Psiquiatria $^{(5)}$.

Estudos realizados com 199 pacientes em internação psiquiátrica, com sintomas graves de depressão, ansiedade e transtornos de personalidade, mostraram a efetividade terapêutica de dois protocolos fisioterápicos, um deles personalizado e baseado em atividades psicomotoras, treino de força e exercícios de resistência aeróbica; e outro que incluía atividades físicas gerais com diferentes formas de exercícios e relaxamentos. Os resultados comparativos entre os dois protocolos mostraram, em ambos, melhora significativa em relação à autoestima, imagem corporal, força muscular, desempenho cardiovascular e melhora nos sintomas de depressão e ansiedade ${ }^{(2,6-7)}$.

Sessões de fisioterapia, incluindo exercícios da terapia de bioenergética, realizadas com portadores de sofrimento psíquico e dependência química em internação psiquiátrica, produziram mudanças favoráveis aos aspectos emocionais e à expressividade verbal. Houve melhora, também, nos sintomas de despersonalização, dores musculares, comprometimentos respiratórios e na sensação de angústia ${ }^{(5)}$.

As contribuições das técnicas de relaxamento e alongamentos, aplicadas em portadores de transtornos mentais usuários de um serviço aberto de assistência psiquiátrica, foram verificadas, e os resultados mostraram melhora nos níveis de ansiedade, aquietação dos pensamentos e ganhos na qualidade do sono ${ }^{(8)}$.

Destaca-se a contribuição de oficinas terapêuticas corporais, fundamentada em técnicas fisioterápicas e de dança, na reabilitação psicossocial e no exercício da autonomia de portadores de transtornos mentais graves e crônicos em hospital dia, enfatizando a promoção da saúde e não da $\operatorname{cura}^{(1)}$.

\section{Objetivo}

Os objetivos deste estudo foram mostrar a contribuição da fisioterapia na minimização do comprometimento corporal proveniente de diversos tratamentos psiquiátricos, e na reabilitação psicossocial de portadores de transtornos mentais.

\section{Método}

O presente estudo é de natureza descritivaexploratória e abordagem qualitativa, que permite o estudo das relações, representações, crenças, percepções e opiniões, produtos das interpretações que os indivíduos fazem do seu modo de viver, sentir e pensar. Essa abordagem é entendida como mais adequada aos estudos de grupos, em que se pretende captar a visão dos sujeitos frente aos acontecimentos sociais ${ }^{(9)}$

Os resultados foram apresentados de forma descritiva e analisados à luz do interacionismo simbólico e do conhecimento científico produzido na área da Fisioterapia. A escolha do interacionismo simbólico como referencial teórico-metodológico deu-se pelo interesse de se aprofundar o estudo dos modos como os sujeitos utilizaram o corpo para se expressar nos grupos sociais, e como aconteceram as interações entre os participantes durante a atividade proposta ${ }^{(10-11)}$.

Sujeitos e local

Para os objetivos do presente estudo, foram convidados a participar, como sujeitos, todos os portadores de transtornos mentais que frequentavam um centro de atenção psicossocial (CAPS), nos dias e horários da semana em que a atividade fisioterapêutica era realizada, sendo que essa atividade oferecia condições para isso, em termos de número de participantes e local para seu desenvolvimento. A partir do aceite dos possíveis sujeitos, as sessões de fisioterapia passaram a ser contadas para os propósitos da pesquisa, e foram estabelecidas 24 sessões para esse fim, com um tempo de três meses para sua realização. Foram inclusos, definitivamente, no estudo os portadores de transtornos mentais que frequentaram até a última sessão e tiveram presença em 20 ou mais sessões, somando, assim, o total de 15 participantes.

Dentre os sujeitos, 12 eram do sexo feminino e 3 do sexo masculino, faixa etária entre 31 e 63 anos, grande variação no estado civil e escolaridade, predominância da religião católica e, quanto aos diagnósticos, destaque para a esquizofrenia, com sete sujeitos, transtorno depressivo recorrente, com cinco sujeitos, sendo, os demais, transtorno dissociativo, de personalidade e bipolar, conferindo à amostra variação em termos de características gerais, importante para as finalidades da pesquisa, por permitir amplitude em termos de possibilidades para a avaliação de seus propósitos.

\section{Procedimentos}

O projeto de pesquisa foi inicialmente examinado e aprovado pelo Comitê de Ética em Pesquisa Envolvendo Seres Humanos da Escola de Enfermagem de Ribeirão Preto da Universidade de São Paulo, Of. CEP-EERP/ USP-119/2008. Os sujeitos foram informados acerca dos procedimentos e da proposta terapêutica da qual iriam participar, tendo sido comunicados de que fariam parte de uma pesquisa. Após as informações, e com o seu aceite para a participação, os sujeitos e seus responsáveis assinaram o termo de consentimento livre e esclarecido. Terminada essa fase inicial, foi iniciada a intervenção terapêutica, fundamentada em técnicas cinesioterápicas, que utilizam o movimento como recurso terapêutico e que tem sua fundamentação no domínio teórico da Fisioterapia, estruturada em 24 sessões de fisioterapia, realizadas duas vezes por semana, traduzindo-se em um 
período de assistência de três meses. As sessões tiveram duração média de duas horas e foram desenvolvidas em espaço apropriado do centro de atenção psicossocial. Ao término de cada sessão, realizou-se uma dinâmica verbal e os participantes deram depoimentos, que foram gravados.

Os recursos fisioterápicos utilizados nas sessões foram exercícios cinesioterápicos, técnicas de consciência e expressão corporal, exercícios posturais, massagem terapêutica, relaxamentos e dinâmicas de interação.

A dinâmica verbal foi conduzida pela própria pesquisadora e orientada por um roteiro de perguntas, com o objetivo de incentivar os participantes a falarem sobre suas percepções referentes às atividades corporais: 1) Quais suas sensações corporais neste momento? 2) Quais sentimentos foram despertados em você durante a sessão? 3) Como estava seu corpo quando chegou e como está agora? 4) Descobriu alguma coisa nova sobre seu corpo ou sobre seus sentimentos hoje? 5) Sentiu dificuldades em alguma atividade?

O planejamento e compilação das estratégias terapêuticas, para a parte interventiva deste estudo, fundamentaram-se em pesquisa bibliográfica, acerca dos recursos terapêuticos considerados adequados à proposta do estudo.

Os objetivos das sessões de fisioterapia foram estabelecidos de acordo com o levantamento bibliográfico acerca dos comprometimentos corporais mais frequentes, em portadores de transtornos mentais usuários de centros de atenção psicossocial, e em consonância com as necessidades específicas do grupo, apontadas pela equipe multiprofissional do centro onde se desenvolveu o presente estudo.

Assim, foram delimitados os seguintes objetivos para as sessões: amenizar os sintomas dos transtornos do movimento provocados por efeito adverso de medicação, melhorar a funcionalidade motora, promover relaxamento, desenvolver consciência e expressão corporal, estimular o contato corporal e a interação entre os participantes.

Por ser esta uma pesquisa que se propôs a investigar uma intervenção terapêutica no contexto da reabilitação psicossocial e que priorizou a reabilitação psicossoial dos usuários, optou-se pela realização das sessões em grupo e, para isso, as técnicas fisioterápicas empregadas na pesquisa foram adaptadas ao trabalho em grupo.

De acordo com o interacionismo simbólico, cada indivíduo é profundamente modificado pelo grupo, pois, a partir do convívio social, ele cria significados para os objetos de seu mundo pessoal ${ }^{(10-11)}$.

Assim, os grupos terapêuticos ou as oficinas terapêuticas modificam a visão de mundo do portador de transtorno mental, ajudando-o a sair da visão restritiva e do isolamento social provocados pela condição fisiopatológica.

As atividades corporais das sessões foram estruturadas basicamente em seis momentos, quais sejam: interiorização, aquecimento, toque terapêutico, trabalho de imagem corporal, expressão corporal e relaxamento.

1. Interiorização: auxiliar o indivíduo a restabelecer o contato consigo, conduzir os participantes ao hábito de perceber e sentir seu corpo ${ }^{(12-13)}$.

2. Aquecimento: preparar o corpo para executar os exercícios físicos, ativando as funções do organismo e prevenindo lesões ${ }^{(14)}$. Desenvolvido através de exercícios cinesioterápicos para coordenação motora, equilíbrio, alongamentos e fortalecimento muscular. Nesta fase também foram realizados exercícios para correção postural, dinâmicas de movimento espontâneo e dinâmicas de integração grupal.

3. Toque terapêutico: minimizar a dificuldade para estabelecer contato físico, promover corpos mais livres de bloqueios e mais preparados para o convívio social ${ }^{(15)}$. Foram utilizadas técnicas de massagem em duplas, massagem em grupos, automassagem e dinâmicas de contato corporal.

4. Imagem corporal: a imagem corporal de portadores de transtornos mentais reflete um mundo interno marcado pela cisão do psiquismo, acentuada indiferenciação afetiva e intelectual ${ }^{(16-17)}$. A imagem corporal foi trabalhada em todas as atividades da oficina, através do movimento, da correção postural, da interação grupal, da expressividade e da interiorização. Contudo, foi uma atividade especialmente realizada através de dinâmicas com espelho.

5. Expressão corporal: permite o despertar do corpo a partir de três níveis: a) ensina a tomar posse do corpo reconhecê-lo, em suas possibilidades e em suas limitações; b) ensina a exprimir sensações através do movimento; c) ensina e promove o encontro com o outro, facilitando a comunicação verbal e a interação social ${ }^{(18)}$.

6. Relaxamento: diminuir tensões físicas, despertar sensações de quietude mental e leveza corporal, promover a autovalorização, estimular a serenidade, paz interior, confiança nas outras pessoas e em si mesmo ${ }^{(19)}$. Permite também reforçar os conteúdos trabalhados durante a sessão. As técnicas utilizadas foram relaxamento de Jacobson e técnica das imagens mentais.

\section{Resultados e Discussão}

A fisioterapia contribuiu para a reabilitação psicossocial dos sujeitos deste estudo, minimizando os comprometimentos corporais causados pelos transtornos mentais, promovendo benefícios físicos e psíquicos. Favoreceu a interação e a convivência entre os usuários, estimulou as relações interpessoais e a expressividade.

Os benefícios físicos estiveram relacionados ao alívio de dores, melhora da funcionalidade motora, motivação, disposição física e as atividades diárias passaram a ser realizadas com mais facilidade. As falas dos sujeitos, denominados $S$, ilustram esses aspectos. Agora tenho mais rapidez nos movimentos e meu corpo está mais condicionando, melhorou as dores e a postura. Eu me tornei uma pessoa mais ágil, mais forte, sem medo (S13). Contribuiu para o meu corpo ficar mais leve, mais relaxado e pronto para as outras atividades. Estou sentindo mais motivada a vir para o CAPS (S3). Melhorou o condicionamento físico e o equilibrio, os exercícios também me ajudaram a ficar mais relaxada para dormir, deu até pra diminuir a medicação pra dormir (S15). Eu estou mais animada, antes eu tinha um desânimo que eu não conseguia melhorar. Agora eu comecei a ficar animada, limpar a casa, fazer comida, 
antes eu não fazia (S5). Depois que eu estou fazendo esses exercícios aqui, estou voltando a fazer minhas tarefas em casa, antes eu estava muito prostrada e com dor, agora melhorou bastante (S7). Eu me tornei mais flexivel, mais leve, com mais ânimo, disposição, sensação de bem-estar, entusiasmo. E isso ai fez sentir meu corpo jovem... Mulher (S13).

As terapêuticas corporais promovem a autopercepção, permitindo ao indivíduo redimensionar atitudes, reconhecer necessidades e transformar a qualidade da própria vida ${ }^{(12)}$.

A independência funcional representa outro aspecto fundamental na reabilitação de portadores de transtornos mentais, conforme a fala a seguir. Pra andar na rua, à noite, era uma dificuldade, sentia muita tontura e caía muito. Hoje eu saio sozinha, até à noite (S1).

Os benefícios psíquicos alcançados estiveram relacionados à motivação, alívio da ansiedade e agitação mental, melhora da autoestima e relaxamento, conforme depoimentos que seguem. O exercício físico gera saúde fisica e mental, a cabeça fica mais aliviada, mais preparada para o raciocinio e ajuda a gente fazer outras atividades (S3). Despertou esperança em coisas boas, esperança de melhorar e vontade de trabalhar (S4). Acabou um pouco a timidez. Eu sinto mais animada, mais comunicativa (S5). Melhorei muito meus pensamentos, eu tinha vontade de suicidar e sair andando sem rumo, agora eu não tenho estes pensamentos mais, tenho pensamentos mais positivos (S11). Mudou, estou com a cabeça melhor, a memória melhor. Eu acho a vida boa, não tenho mais vontade de morrer, eu tenho é vontade de viver e viver bem (S13). Ajudou bastante a não pensar demais. Quando vou dormir eu lembro de fazer o relaxamento e hora que eu vejo já amanheceu. Também ajudou na relação pessoal, a conversar. Nem conversar eu não gostava (S15). Ajudava a maneirar a minha mente, aliviar a cabeça quente e pesada. Ela ficava leve, ficava como se tivesse tomado um calmante. Melhorava a cabeça e o corpo ao mesmo tempo (S6).

Percebe-se que, ao colocar a atenção em seu corpo, o indivíduo abre a possibilidade de comunicação entre sua mente e seu corpo, tornando possível vivenciar os movimentos e ressignificar a consciência corporal, seus modos de pensar, agir e sentir ${ }^{(1)}$.

Favoreceu a interação e a convivência entre os usuários e estimulou as relações de amizade, tornando-os mais receptivos para se relacionarem e se expressarem, conforme os depoimentos a seguir. $O$ melhor foi a convivência, foi muito bom ficar no meio de muitas pessoas, a gente se abre mais, tem mais confiança de conversar (S1). O encontro com o outro ajuda a gente a se encontrar também (S3). Fica a amizade e o afeto porque antes a gente era travada pra ter um gesto de carinho com as pessoas, foi tornando a minha vida mais agradável (S13). Junto com os colegas, um apoia o outro, um precisa do outro para fortalecer (S7).

Neste estudo, as interações grupais foram aprimoradas entre os participantes durante as sessões e favoreceram a socialização, expressividade e comunicação, autoestima, afetividade, companheirismo, solidariedade, sensação de acolhimento e confiança em outras pessoas. Para alguns sujeitos, a vivência desse aprendizado se estendeu ao convívio familiar, conforme mostram os depoimentos de S15, S11 e S13. Mudou com a minha filha, ela ficou mais carinhosa, eu mudei (S15). Eu quase não conversava com a minha filha e agora a gente conversa (S11). Eu estou convivendo mais com a minha filha (S13).

Os sujeitos tornaram-se mais receptivos ao contato físico, houve experiências importantes relacionadas à afetividade e à convivência, desenvolveram o aprendizado da confiança e o aprofundamento das relações de amizade. Os depoimentos que seguem mostram esses aspectos. $A$ massagem com os pés foi o que mais me marcou. Eu senti contato com o ser humano que eu não conseguia sentir antes porque eu não conseguia que ninguém me encostasse. Naquele momento eu consegui entregar o meu corpo (S8). Aquele exercício de toque, massagem, relaxamento, foi bom porque a gente sente carente, então a gente toca nas pessoas aí desperta uma emoção (S6). Eu senti que estava fazendo carinho nas pessoas. Eu sinto falta disso, sinto sozinha. Senti vontade de namorar, foi carinhoso, sentimento bom (S5). O convívio de grupo traz paz, amor, saúde, esperança de vida, calor, solidariedade, sensação de proteção (S6). Fez a gente sentir o contato com a pessoa, sentir que está sendo compreendida sem falar nada, só com o contato (S7).

Perceber a importância do contato físico é um aspecto fundamental para o portador de sofrimento físico, para que seja atenuado o distanciamento em que, com frequência, eles se colocam ${ }^{(18)}$.

O toque terapêutico despertou sentimento de pertença grupal e trouxe à consciência conteúdos relevantes à reestruturação dos aspectos afetivos e emocionais no processo de reabilitação, ilustrado pelo depoimento apresentado a seguir. Mudou a confiança que a gente tem, antes tinha medo e vergonha de tocar na pessoa, de cumprimentar, de abraçar... Ai foi mudando e abriu a mente, ficou mais fácil. Agora, qualquer um que entra aqui eu já puxo conversa (S1).

Houve melhora nos aspectos referentes à imagem corporal, autoestima e aceitação do próprio corpo, confirmados pelos depoimentos que seguem. Trouxe mais autoestima, eu me sentia feia e melhorou bastante. Eu passei a ver que eu não sou tão feia assim como eu pensava. Eu me olhei no espelho e consegui me admirar um pouco, não me senti tão ruim como eu sentia antes (S7). A aceitação do corpo melhorou (S15). Achei que eu estou até parecendo gente, mais bonita (S10). Vontade comprar um vestido colorido, vontade me sentir mulher (S3).

O trabalho corporal é capaz de suscitar alterações no estado psicológico, por desenvolver uma nova percepção da imagem corporal, promovendo mudanças no comportamento, autoestima e humor ${ }^{(20)}$.

As limitações na capacidade de expressão corporal dos portadores de transtorno mental, gestos limitados e movimentação restrita impedem que se expressem com eficiência para realizar uma boa comunicação social ${ }^{(1,18)}$.

A expressividade mostrou-se comprometida pela somatória de fatores: distanciamento do corpo, desinteresse pelos cuidados pessoais, baixa autoestima, rejeição à própria imagem e alterações corporais decorrentes do transtorno mental. Para promover a expressão corporal foi fundamental atenuar as alterações na corporeidade, decorrentes dos efeitos adversos da medicação.

As alterações corporais mais referidas pelos sujeitos foram: lentidão de movimentos, rigidez corporal, dificuldade para relaxar, indisposição, desânimo, fraqueza, cansaço, dores no corpo, dificuldades de marcha, tontura, tremor, quedas, dificuldade na realização de atividades diárias e robotização dos movimentos, alterações essas presentes nos depoimentos de S1, S3, S7 e S11. Sinto dor no corpo, dor nos ossos e cansaço. Eu sinto a cabeça muito ruim, com muita tontura, quase não paro em pé (S1). Tenho tremor e 
a minha escrita está horrivel, não consigo firmar a caneta (S3). Estou muito parada (S7). Eu estou que nem um robô (S11).

Sintomas que comprometem o corpo e o movimento aparecem com frequência após o uso de drogas antipsicóticas, medicamentos inibidores das funções psicomotoras que podem provocar efeitos adversos, especialmente relacionados aos transtornos de movimento ${ }^{(4)}$, assim descritos no depoimento de S15. Os meus movimentos estão lentos, a minha perna ficou dura, enrijeceu, parecendo um robozinho. Está tão duro e tão pesado que eu não consigo andar direito. Eu não consigo relaxar, fico tensa. Os músculos estão doendo e está tudo tenso. Por estar fazendo tudo lento, atrapalha arrumar um emprego, atrapalha arrumar um namorado, atrapalha sair de casa (S15).

As dificuldades de inserção social do portador de transtorno mental estão relacionadas a esses comprometimentos corporais ${ }^{(1,18)}$. A fisioterapia mostrou que pode interromper esse processo, auxiliando o portador de transtorno mental a criar um corpo mais funcional e expressivo, capaz de facilitar sua interação social.

Os sujeitos adquiriram maior proximidade com seus corpos e aprimoraram sua capacidade de livre expressão, conforme os depoimentos a seguir. Que bom a gente poder se soltar! Despertou esse saco do riso que há muito tempo estava guardado lá dentro. É a criança dentro de nós que está vindo, alegre, brincando. Fazendo exercício, brincando (S3). Eu nunca tinha conseguido me soltar perto de outras pessoas, e com vocês eu consegui. Ajudou a me soltar mais (S8).

A expressão corporal confere autonomia e liberdade, despertando para a possibilidade de se projetar efetivamente no mundo à sua volta ${ }^{(13)}$. Esse encontro consigo mesmo e a reconstrução da individualidade vislumbra ao portador de transtorno mental a possibilidade de uma verdadeira inserção na sociedade. Os depoimentos descritos abaixo revelam isso. Eu não sabia que fisioterapia era isso também... Pensava que fosse aquela coisa mecânica, um monte de exercícios. Então eu vi que juntava tudo: os exercícios, com a música e com a imaginação. Trabalhou tudo, o corpo por completo $(\mathrm{S} 3)$.

As experimentações investigadas neste estudo buscaram provocar no portador de transtorno mental um despertar para o próprio corpo e para a possibilidade de livre expressão corporal, utilizando como recurso as técnicas fisioterápicas. Buscou mostrar aos sujeitos um novo olhar para seu corpo, para o outro e para o mundo, tornando essas descobertas um instrumento facilitador da sua reabilitação.

O interacionismo simbólico, utilizado como fundamentação teórica deste estudo, permitiu compreender como o grupo afetou cada indivíduo nos aspectos relacionados à reabilitação psicossocial, como aconteceram as interações entre os participantes, quais as repercussões desses fatores para a sua reabilitação psicossocial, e evidenciou que a compreensão que os portadores de transtorno mental têm de si mesmos e de seu corpo é aguçada pelo fator grupo.

Segundo os relatos dos sujeitos, a realização das atividades em grupo favoreceu a interação entre os participantes, promovendo o sentimento de grupalidade, a sensação de acolhimento e confiança em outras pessoas, tornando-os mais receptivos para se relacionarem.
A interação do grupo também favoreceu o desenvolvimento da expressividade e comunicação dos participantes, evidenciando que, quando o indivíduo se sente pertencente a um grupo, sua autoestima é fortalecida e ele se torna mais confiante para se envolver em novas relações sociais, configurando fator relevante para a reabilitação psicossocial.

\section{Conclusão}

A participação da equipe multiprofissional do centro de atenção psicossocial, onde foi desenvolvido o presente estudo, na indicação dos sujeitos, foi extremamente importante, pois, selecionando usuários com características gerais diversas, possibilitou a intervenção da fisioterapia de maneira ampla, atingindo pessoas com diferentes comportamentos frente aos seus transtornos mentais e possibilidades de respostas variadas. Foi também extremamente importante a escolha do local, pois, em se tratando de um centro de atenção psicossocial, é de entendimento que deve estar inserido totalmente no contexto da reforma psiquiátrica e, assim sendo, buscando principalmente a reabilitação psicossocial do usuário que assiste. Portanto, um cenário ideal para as finalidades desta pesquisa.

Nas sessões de fisioterapia, o indivíduo descobriu suas capacidades de expressão e movimento, modificou favoravelmente sua autoimagem e, como consequência, aprendeu a se valorizar como pessoa.

Os recursos fisioterápicos promoveram a saúde mental porque aliviaram a sensação de agitação mental, desaceleraram os pensamentos, melhoraram a disposição, a sensação de leveza física e mental, a concentração, promoveram motivação para participar de outras atividades, para interagir socialmente e para se relacionar com outras pessoas, trazendo alegria e melhorando a autoestima.

Assim sendo, o estudo mostrou que a fisioterapia foi capaz de minimizar os comprometimentos corporais causados pelos transtornos mentais. Contribuiu para a reabilitação psicossocial, porque auxiliou os portadores de transtornos mentais, promovendo benefícios físicos e psíquicos relacionados ao alívio de dores e ansiedade. Promoveu, também, a melhora da função motora, da autoestima, do ânimo e da disposição, favorecendo, ainda, a interação e a convivência entre os usuários, estimulando as relações de amizade, tornando-os mais receptivos para se relacionar e se expressar.

As sessões de fisioterapia representaram também um espaço para os sujeitos perceberem e elaborarem, através das dinâmicas verbais, os avanços conquistados em seu processo de reabilitação, dando-lhes a possibilidade de sonhar com novas coisas e ressignificar a vida.

A inserção do fisioterapeuta na promoção de saúde mental e na reabilitação psicossocial, em muitos lugares, ainda é uma perspectiva, e, em outros, uma realidade isolada. Espera-se que a atuação do fisioterapeuta, nesse campo, se torne cada vez mais crescente e fecunda, e que a estrutura curricular dos cursos de Fisioterapia priorizem 
também as disciplinas relacionadas à saúde mental, para que os profissionais fisioterapeutas estejam ainda mais capacitados a atuar nessa área, pois, como mostrado neste estudo, sua atuação em equipes de saúde mental, buscando a reabilitação psicossocial do portador de transtorno mental, é particularmente importante.

\section{Referencias}

1. Tessitore EC. Talentos do corpo: uma experiência de trabalho corporal com pacientes com transtorno mental [dissertação de mestrado]. São Paulo (SP): Escola de Enfermagem da Universidade de São Paulo; 2006. 128 p.

2. Furtado JP. A fisioterapia na saúde mental. Rev Fisioter Mov. 1995;8(1):13-24.

3. Sadock BJ, Sadock VA. Compêndio de psiquiatria: ciência do comportamento e psiquiatria clínica. Porto Alegre (RS): Artmed; 2007.

4. Giacon BCC, Galera SAF. Primeiro episódio da esquizofrenia e assistência de enfermagem. Rev Esc Enferm USP. 2006;40(2):286-91.

5. Munhos COM. Atuação fisioterápica em pacientes com transtornos mentais. Rev Psiquiatr Clin. 1996;23(3):11623.

6. Knapen J, Vliet PVan de, Coppenolle HVan, David A, Peuskens J, Pieters G, et al. Comparison of changes in physical self-concept, global self-esteem, depression and anxiety following two different psychomotor therapy programs in nonpsychotic psychiatric inpatients. Psychother Psychosom. 2005;74(6):353-61.

7. Knapen J, Van de Vliet P, Coppenolle H Van; David A, Peuskens J, Knapen K, et al. Of two psychomotor therapy programmes on physical fitness and physical self-concept in nonpsychotic psychiatric patients: a randomized controlled trial. Clin Rehabil. 2003; 17(6):637-47.

8. Marinho LCP, Miolo SB. Ação fisioterápica às necessidades do portador de sofrimento psíquico. Rev Fisioter Mov. 1997;10(2):44-52.

9. Minayo MCS. O desafio do conhecimento: pesquisa qualitativa em saúde. São Paulo: Hucitec; 2006.

10. Blumer H. Interactionism symbolic: perspective and method. Englewood Cliffs: Prentice Hall; 1969.

11. Charon JM. Symbolic interactionism: an introduction, an interpretation, an integration. Englewood Cliffs: Prentice Hall; 1995.

12. Diefenbach N. O eu corporal em terapia morfoanalítica. Rev Fisioter Mov. 2003; 16(2):73-82.

13. Brikman L. A linguagem do movimento. São Paulo: Summus; 1989.
14. Alter MJ. Ciência da flexibilidade. Porto Alegre: Artmed; 1999.

15. Montagu A. Tocar, o significado humano da pele. São Paulo: Summus; 1988.

16. Schilder P. A Imagem do corpo: as energias construtivas da psique. São Paulo: Martins Fontes; 1994.

17. Santos MA. A representação de si na esquizofrenia através do psicodiagnóstico de Rorschach. Paidéia. 2000;10(19):67-81.

18. Pankow G. O homem e sua psicose. Campinas: Papirus; 1989.

19. Elias ACA, Giglio JS, Pimenta CAM, El-Dash LG. Programa de treinamento sobre a intervenção terapêutica "relaxamento, imagens mentais e espiritualidade" (RIME) para re-significar a dor espiritual de pacientes terminais. Rev Psiquitr Clin. 2007;34(1): 60-72.

20. Lederman E. Fundamentos da terapia manual. São Paulo: Manole; 2001.

\section{Como citar este artigo:}

Silva SB, Pedrão LJ, Miasso AI. O impacto da fisioterapia na reabilitação psicossocial de portadores de transtornos mentais. SMAD, Rev. Eletrônica Saúde Mental Álcool Drog. (Ed. port.). jan.-abr. 2012 [acesso:

40. Disponível em: 